\title{
INADVERTENT COMPLICATION OF PROSTHETIC VALVE SURGERY: LEAFLET PERFORATION
}

\author{
Mehmet Dogan, Sadik Acikel, Ugur Arslantas, Tolga Cimen, Ekrem Yeter
}

Ministry of Health Diskapi Yildirim Beyazit Research and Educational Hospital, Department of Cardiology, Ankara, Turkey

Summary: There are various complications of prosthetic valvular surgeries. Among them, leaflet perforation should be emphasized and brought to mind when there have been eccentric valvular regurgitation. In this report, we presented 2 cases of iatrogenic aortic and mitral valve leaflet perforation after prosthetic valve surgeries.

Key words: Mechanical heart valve; Complication; Leaflet perforation

\section{Introduction}

Aortic or mitral regurgitation caused by a leaflet perforation is most frequently associated with infective endocarditis that involves the aortic or mitral valve. However, the suture-related injury to an aortic or mitral valve can result in leaflet perforation with aortic or mitral regurgitation after such perivalvular surgeries (1-4). Herein, we reported 2 cases of iatrogenic aortic and mitral valve leaflet perforation after prosthetic valve surgeries.

\section{Case 1}

A 50-year-old female patient was admitted to our department with complaint of exertional dyspnea. Her past medical history revealed an aortic valve replacement in 1997. On 2008 the patient was re-operated for patient-prosthesis mismatch and a permanent pacemaker was implanted after the operation for complete atrioventricular block. There was no any history of infective endocarditis or trauma. On physical examination, blood pressure was 100/60 mmHg and heart rate was 100 bpm. Physical examination revealed $3 / 6$ systolic murmur and metallic mitral valve clicks at the apex. Electrocardiography (ECG) revealed paced rhythm. Biochemistry panel and hemogram parameters were within normal limits and INR was 2.6. Transthoracic echocardiography (TTE) showed that left ventricular diameters and systolic functions were normal. Prosthetic aortic valve was functional (maximum $17 \mathrm{mmHg}$ systolic gradient) and the left atrium was dilated. Right

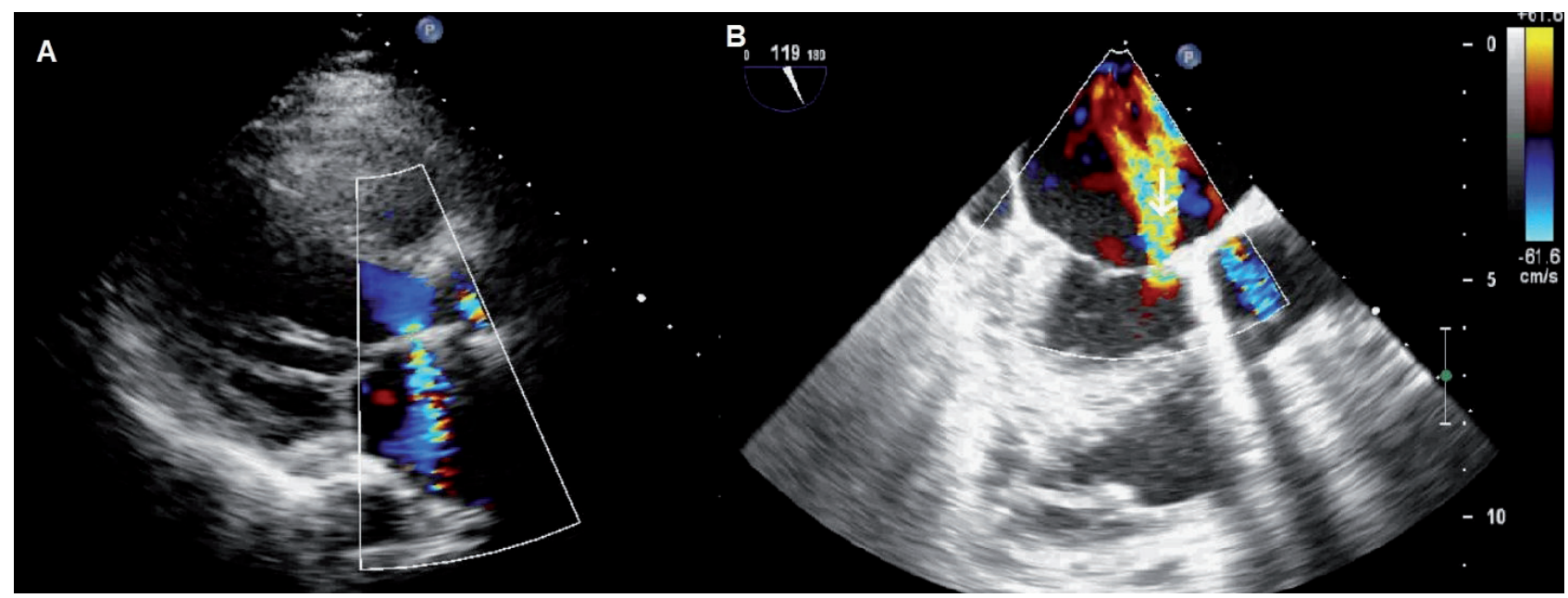

Fig. 1: (A) Color Doppler echocardiography in the parasternal long axis view showing significant regurgitant jet from left ventricle to left atrium. (B) Transesophageal echocardiography view showing significant regurgitant jet from left ventricle to left atrium (arrow). 


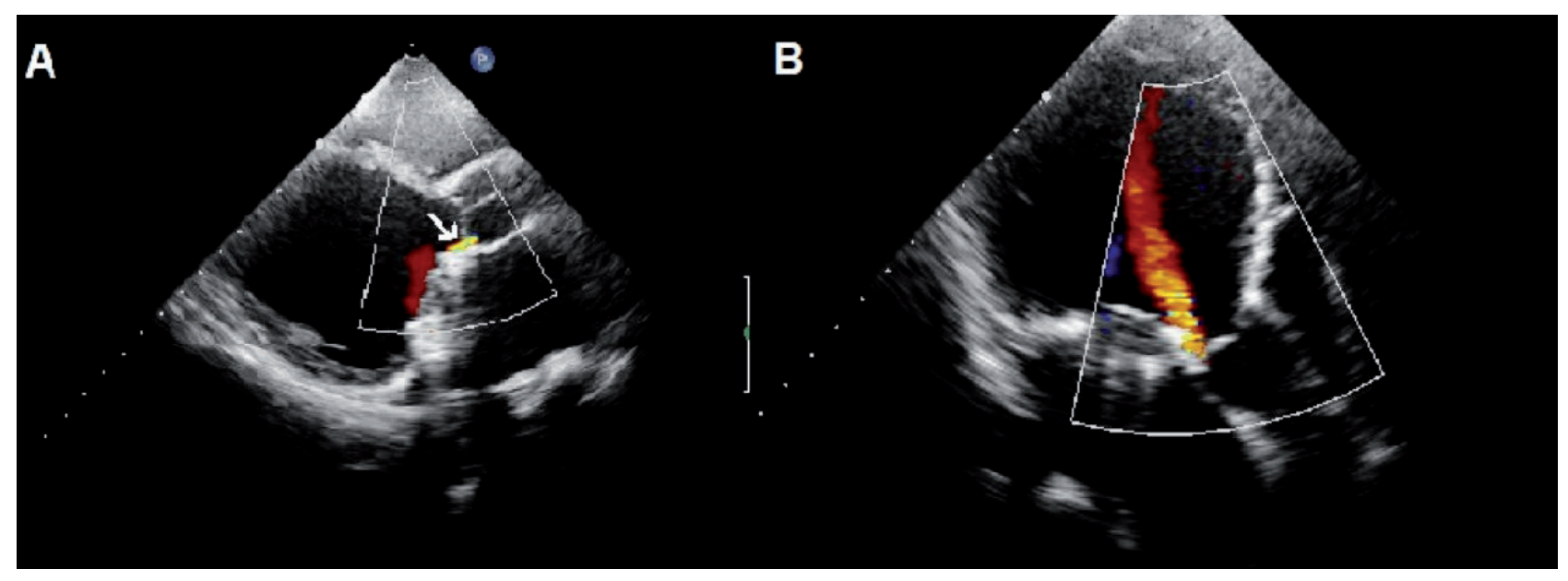

Fig. 2: (A) Color Doppler echocardiography in the parasternal long axis view showing a mild aortic regurgitation jet at basal part of non-coronary cusp of the aortic valve (arrow). (B) Color Doppler echocardiography in the apical five-chamber view showing a mild aortic regurgitation.

cardiac chambers were also dilated with moderate tricuspit regurgitation (TR) and systolic pulmonary artery pressure was $50 \mathrm{mmHg}$. TTE demonstrated a perforation adjacent to mitral-aortic intervalvular fibrosa region on anterior mitral leaflet (Fig. 1A). Color Doppler examination revealed significant regurgitant jet from left ventricle to left atrium in that perforated area (Fig. 1B). On 2D plane images the radius of the perforated area was $0.5 \mathrm{~cm}$. Patient was referred for mitral valve surgery. But because of the patient has refused the operation, she was followed up with medical therapy.

\section{Case 2}

A 30-year-old male patient was admitted to our hospital for routine control visit. Prosthetic mitral valve replacement was performed for rheumatic mitral regurgitation 10 years ago. Also, because of development of non-ischemic cardiomyopathy at follow-up, ICD implantation was performed 1 year ago for primary prevention. He has no history of infective endocarditis or trauma. TTE revealed dilated left ventricular diameters left ventricular ejection fraction of $30 \%$. Prosthetic valve on mitral position was functional. On parasternal long axis and apical-4-chamber views, there was a perforated region at the basal part of non-coronary cusp of the aortic valve. Color Doppler investigation revealed a mild aortic regurgitation at that region (Fig. 2). Patient was followed-up with medical therapy.

\section{Discussion}

Prosthetic valve surgeries may result in various complications both at early and late postsurgical follow-up. Leaflet perforation is a very rarely seen phenomenon in this regard. It most frequently occurs due to infective endocarditis, but it can also be occurred iatrogenically during peri-valvular surgeries (1-3). Surgical forceps or needle injury of the leaflets are among the causative insulting factors (4).

It has been reported previously that iatrogenic aortic valve perforation might develop after cardiovascular interventional and surgical procedures like mitral valve replacement, ventricular or atrial septal defect repair and after radiofrequency ablation (2-7). Iatrogenic aortic valve injury is most often a leaflet tear or perforation. Iatrogenic injury to aortic valve may occur at any of the three leaflets which depends on the surgery type. Such as, noncoronary cusp is more prone to injuries during mitral or aortic valve surgeries and atrial septal defect operation. Left coronary leaflet is more prone to injuries during mitral valve operation $(5,6)$ and right coronary cusp may be injured during ventricular septal defect operation and pulmonary valve operation $(2,8)$.

To our knowledge, there was no data regarding iatrogenic mitral valve perforation after aortic valve replacement in the literature. In this regard, our case is the first in the literature. This can be due to the paucity of cases, also these cases can be unrecognized or underreported. We thought that during re-operation for patient-prosthesis mismatch, mitral valve could be damaged more easily due to the severe adhesions.

In conclusion, evaluation of patients after valvular surgery, especially in whom a new murmur is detected, should include a careful echocardiographic examination. When there was an eccentric regurgitant jet, leaflet perforation should bear in mind and a detailed evaluation should be done accordingly. These case reports stress on the importance of intraoperative TEE in confirming the adequacy of valvular surgery as well as ruling out possible iatrogenic damage to adjacent structures. A thorough examination of contiguous structures is essential. 


\section{References}

1. Ballal RS, Mahan EF 3rd, Nanda NC, et al. Aortic and mitral valve perforation: diagnosis by transesophageal echocardiography and Doppler color flow imaging. Am Heart J 1991; 121: 214-17.

2. Woo JJ, Koh YY, Chang KS, et al. A case of iatrogenic aortic valve leaflet perforation after closure of a ventricular septal defect. Int J Cardiovasc Imaging 2010; 26 Suppl 1: 169-72.

3. Zhang T, Jiang S, Wang Y, et al. Surgery on a patient with iatrogenic aortic valve leaflet perforation after repair of a congenital ventricular septal defect. Heart Surg Forum 2013; 16(2): E103-6.
4. Rey C, Vaksmann G, Brevière GM, et al. Aortic valve insufficiency: an unrecognized complication of the surgical repair of ostium primum atrial septal defect. Arch Mal Coeur Vaiss 1991; 84(5): 627-31.

5. Rother A, Smith B, Adams DH, et al. TEE diagnosis of acute aortic valve insufficiency after mitral valve repair. Anesth Analg 2000; 91: 499-500.

6. Mehta AR, Hunsaker R. Iatrogenic aortic incompetence after mitral valve replacement. J Cardiothorac Vasc Anesth 2007; 21(2): 276-8.

7. Fisch-Thomsen M, Jensen JK, Egeblad H, et al. Mitral valve perforation appearing years after radiofrequency ablation. J Heart Valve Dis 2011; 20(3): 351-2. 8. Hill AC, Banel RC, Razzouk AJ et al. Echocardiographic recognition of iatrogenic aortic valve leaflet perforation. Ann Thorac Surg 1997; 64(3): 684-89.

Received: $25 / 05 / 2013$

Accepted in revised form: 23/10/2013

\section{Corresponding author:}

Mehmet Dogan, MD, Ministry of Health Diskapi Yildirim Beyazit Research and Educational Hospital, Department of Cardiology, 06110 Ankara, Turkey; e-mail: drmehmetdogan@yahoo.com 